\title{
Plug \& Produce im real-virtuellen Kontext fertigungstechnischer heterogener Anlagen
}

\section{Steuerungsarchitektur und Virtuelle Inbetriebnahme}

\author{
P. Habiger ${ }^{1}$, G. Hildebrandt ${ }^{2}$, R. Drath ${ }^{3}$, M. Barth ${ }^{4}$, A. Fay ${ }^{5}$, A. Zor ${ }^{6}$ und M. Marseu ${ }^{7}$
}

\section{Zusammenfassung}

Die Wandelbarkeit von Anlagen im Kontext der Industrie 4.0 führt dazu, dass zukünftig Fertigungsmodule unterschiedlicher Hersteller interagieren müssen - es entsteht eine heterogene Modullandschaft. Das erfordert Lösungen für die automatisierungstechnische Einbindung und den flexiblen Austausch von weiteren, herstellerfremden und bislang unbekannten Modulen. Es werden Lösungskonzepte zur Umsetzung eines strukturierten und herstellerunabhängigen Engineering-Konzepts für Plug \& Produce in einer heterogenen Landschaft von Fertigungsmodulen benötigt. Im Rahmen dieser Arbeit stellen die Autoren eine Architektur vor, wie dieses Ziel erreicht werden kann. Der Fokus liegt dabei auf der Steuerungsarchitektur, der Informationsmodellierung und der Virtuellen Inbetriebnahme als Grundlage für das gemischt real-virtuelle Engineering. Das vorgestellte Konzept basiert auf einer Anforderungsanalyse und ist prototypisch umgesetzt.

\section{Stichwörter}

Steuerungsarchitektur, Virtuelle Inbetriebnahme, Plug \& Produce, Modulare Automation

\section{Motivation und Zielstellung}

Die steigende Kundennachfrage nach individuellen Produkten erhöht die Anzahl der Produktvarianten und verkürzt damit die Dauer der Produktlebenszyklen [1]. Dies führt zu einer steigenden Komplexität und kürzeren Entwicklungszeiten im Anlagenbau. Folglich muss der Engineering-Prozess vereinfacht, kostengünstiger und effizienter werden [2]. Zwei wirkungsvolle Ansätze, die diese Ziele verfolgen, sind die Modularisierung von Produktionsanlagen [3] und das virtuelle Engineering [4]. Virtuelles Engineering führt die Idee der Virtuellen Inbetriebnahme (VIBN) in die früheren Phasen des Engineerings ein. Während in der VIBN simulative Methoden am Ende des Engineeringprozesses für Testzwecke verwendet werden, werden sie bei virtuellem Engineering von Beginn an über den gesamten Entwicklungszeitraum und darüber hinaus verwendet. Es ermöglicht frühzeitige Tests und

\footnotetext{
${ }^{1}$ Pascal Habiger, Hochschule Pforzheim, Wissenschaftlicher Mitarbeiter

${ }^{2}$ Gary Hildebrandt, Hochschule Pforzheim, Wissenschaftlicher Mitarbeiter

${ }^{3}$ Prof. Dr.-Ing. Rainer Drath, Hochschule Pforzheim, Prof. Mechatronische Systementwicklung

${ }^{4}$ Prof. Dr.-Ing. Mike Barth, Hochschule Pforzheim, Prof. Engineering mechatronischer Komponenten

${ }^{5}$ Prof. Dr.-Ing. Alexander Fay, Helmut-Schmidt-Universität, Prof. Automatisierungstechnik

${ }^{6}$ Ayhan Zor, Festo Didactic SE, Education DE Regionalleiter Süd-West

${ }^{7}$ Moritz Marseu, Festo Didactic SE, Digital Factory and Smart Automation
} 
verbessert das Verständnis des zu betrachtenden Systems in jeder Entwicklungsphase, ohne dass die entsprechende Hardware physisch vorhanden sein muss. Die Modularisierung umfasst die Zerlegung einer Anlage in standardisierte funktionale Einheiten (Module), die nach dem Baukastenprinzip flexibel und herstellerübergreifend zusammengesetzt werden und interoperabel agieren können. Modulare, industrielle bzw. zu didaktischen Mitteln eingesetzte Fertigungsanlagen wie etwa die CP Factory von Festo Didactic SE agieren mangels einheitlicher verfügbarer Standards üblicherweise im Rahmen vorher vereinbarter und proprietärer Steuerungs- und Kommunikationsstrukturen im homogenen Umfeld aus zueinander passenden Modulen und einer Orchestrierung.

Die Zukunft erfordert jedoch technische Lösungen für heterogene Systeme, d.h. die automatisierungstechnische Einbindung oder den flexiblen Austausch von weiteren, herstellerfremden und bislang unbekannten Modulen. Heute erfordert dies noch tiefe, komplexe, riskante und daher unerwünschte Eingriffe in die Steuerungsprogramme und in die Orchestrierung. Die Wandelbarkeit von Produktionseinrichtungen zielt jedoch darauf ab, eine Anlage flexibel und adaptiv an veränderte Produktionsziele anpassen zu können, auch mit vorab unbekannten Produktionsmodulen unterschiedlicher Hersteller. Die Bewältigung gewünschter Flexibilität in heterogenen Systemen ist bisher nicht gelöst. Zur Lösung dieser Problemstellung verfolgen die Autoren die Kombination der Methodik der modularen Automation mit der des virtuellen Engineerings. Diese Kombination bietet einen großen Mehrwert für ein effizientes Engineering. Dieser Fusion widmet sich das BMBF-geförderte Forschungsprojekt METHODS (Modular Engineering Techniques for HeterOgeneous Discrete Systems) an der Hochschule Pforzheim in Kooperation mit Festo Didactic SE, Festo SE und der Helmut-Schmidt-Universität. Das Ziel des Forschungsvorhabens ist die Entwicklung eines Engineering-Konzepts für Plug \& Produce in einer heterogenen virtuell-realen Landschaft von Fertigungsmodulen. Für die Umsetzung des Plug \& Produces ist es in einem ersten Schritt notwendig zu klären, wie die Steuerungsarchitektur der Module aufgebaut ist, wie die Informationen für die Integration in einen Anlagenverbund bereitgestellt werden und wie sich die Module in einer Virtuellen Inbetriebnahme verhalten. Basierend darauf sind höherwertige Konzepte anwendbar, sodass die Vision des Projektes erreicht werden kann. Dafür werden in diesem Beitrag zuerst im Stand der Technik zwei relevante Konzepte vorgestellt, die als Basis für den Beitrag dienen. In einer anschließenden Anforderungsanalyse werden Anforderungen an die Steuerungsarchitektur, Informationsmodellierung und VIBN erhoben, die im vorgestellten Konzept, in den dazugehörigen Teilkonzepten und in der prototypischen Implementierung berücksichtigt sind.

\section{Stand der Technik}

Die Grundlage für die Untersuchungen bildet das (homogene) modulare und flexible Ausbildungssystem CP Factory ${ }^{8}$ von Festo Didactic SE. Es besteht aus Grundmodulen (GM), die mit Applikationsmodulen (AM) erweiterbar sind. Über Förderbänder transportieren die Grundmodule Teile, die mit Hilfe der Applikationsmodule bearbeitet werden. Die Module sind an ein Manufacturing Execution System (MES) angeschlossen, welches die Prozesse der Module orchestriert und beobachtet. Im Wesentlichen bestimmt ein proprietäres Interaktionsprotokoll zwischen den GM und dem MES die Funktionalität der Anlage. Neben der zyklischen Zustandskontrolle werden zu spezifischen Events, wie bspw. bei dem Erkennen eines Warenträgers, fest definierte Datenpakete in einem Handshake-Verfahren ausgetauscht. Die Datenpakete beinhalten Informationen über die Produkte, die auszuführenden Dienste und vieles mehr. Die Module und das MES müssen in der Lage sein, die versendeten Datenpakete zu interpretieren und umzusetzen. Die Anlage ist so ausgelegt, dass diese in einer homogenen Welt mit Modulen eines Herstellers einwandfrei funktioniert. Die Transportvorgänge in den GM werden nicht als Dienste abgebildet, sondern werden in Absprache mit dem MES von den GM intern individuell ausgeführt. Die AM sind den GM unterlagert, sodass das GM die Signale des angeschlossenen AM an

${ }^{8}$ CP Factory: https://www.festo-didactic.com/de-de/lernsysteme/fabrikautomation-industrie-4.0/ 
das MES vermittelt. Über eine weitere proprietäre Schnittstelle mit einem proprietären Befehlswortschatz interagieren die GM und AM unter Verwendung impliziter Vereinbarungen. Die Integration der GM in das MES und deren Konfiguration findet manuell über eine proprietäre Konfigurationsdatei statt. Die Einbindung herstellerfremder Module ist ohne Wissen der proprietären Schnittstellen und impliziten Vereinbarungen nicht möglich oder vorgesehen. Hier ist ein tiefer Eingriff erforderlich.

Ein neuer Ansatz zur Modularisierung heterogener Systeme etabliert sich aktuell in der Prozesstechnik: das standardisierte Module Type Package (MTP) für die einheitliche Integration von Modulen in modulare Prozessanlagen [5]. Die Besonderheit besteht darin, dass jedes Modul durch ein elektronisches Informationsmodell beschrieben wird, das alle zum Betrieb notwendigen Informationen explizit modelliert. Das Informationsmodell kann dabei in verschiedene Bereiche wie etwa verfügbare Dienste, Bedienbild oder Alarmmanagement unterteilt werden. Bei dem Zusammenstellen von Modulen werden die beteiligten MTPs in ein Orchestrierungssystem importiert, interpretiert und dienen dort als Grundlage für die Ansteuerung und Überwachung der Module sowie ihrer Orchestrierung. Das Informationsmodell spezifiziert eine Schnittstelle, die unabhängig von konkreten Kommunikationstechnologien gilt. Diese Schnittstelle umfasst die Dienste, deren Parameter und die internen Komponenten. Damit ist ein einheitlicher und herstellerneutraler Zugriff auf die Module möglich. Das Konzept des MTP postuliert, dass Module dienstbasiert mit einem einheitlichen Zustandsmodell sowie definierten Befehlen agieren. Dienste sind über Prozeduren in Varianten ausführbar.

Im Bereich des Plug \& Produce existieren weitere Forschungsarbeiten wie z.B. BaSys 4.2 und openMOS, die in METHODS berücksichtigt werden, jedoch in diesem Beitrag nicht verfolgt werden.

\section{Anforderungsanalyse}

Aus dem Stand der Technik und der Vorgabe einer heterogenen Modullandschaft lassen sich Anforderungen an die Steuerungsarchitektur und Informationsmodellierung ableiten, die u.a. Einflüsse auf die VIBN haben (s. Tabelle 1).

A1 - Harmonisierte Zugriffsschnittstelle: Für die Kopplung von heterogenen Modulen ist es wie bei dem MTP notwendig, dass sich alle beteiligten Systeme untereinander verstehen. Dies kann nur dann funktionieren, wenn sie wörtlich dieselbe Sprache sprechen. Die Voraussetzung dafür ist die Spezifikation einer herstellerneutralen und einheitlichen Zugriffsschnittstelle.

A2 - Dienstbasierte Steuerungsarchitektur: Damit Module austauschbar und die Anlagen wandelbar sind, ist ein einheitlicher Umgang mit den Fähigkeiten und den Funktionalitäten der Module als bspw. Dienste in einer serviceorientierten Architektur wie bei dem MTP notwendig. Die CP Factory setzt zwar Dienste ein, diese haben aber keine spezifizierten Zustände und Befehle und werden nur für bearbeitende Prozesse eingesetzt. Damit die gesamten Funktionalitäten gleich abgebildet werden, sollen alle Arten von Prozessen wie Transport- und Bearbeitungsprozesse als Dienste mit einem einheitlichen Zustandsmodell abgebildet werden.

A3 - Dienstvarianten: Dadurch, dass sämtliche Prozessarten über Dienste ausführbar sind (A2), sollen die Module mehrere Dienste gleichzeitig ausführen können und dabei mit Produktvariationen umgehen können. Damit ist es auch möglich, dass die Dienste hierarchisiert werden, um Kombinationen von Fähigkeiten zu bilden. Damit nicht ähnliche Funktionalitäten von Modulen in mehrere unterschiedlichen Dienste gebündelt werden, sollen Ausführungsvarianten verfügbar sein. Die Anforderungen sind seitens des MTP erfüllt. Die CP Factory erfüllt diese Anforderungen bisher nicht.

A4 - Keine Modulhierarchie: Hierarchien von Modulen haben den Vorteil der Abstraktion und der Bündelung zusammengehöriger Funktionalitäten gegenüber einem Orchestrierungssystem. Für die Hierarchisierung, wie sie bspw. bei der CP Factory realisiert ist, müssen die Module vorher über potenzielle koppelbare Module Kenntnis haben. Damit dies vermieden wird, sollen alle Module unabhängig von deren Prozessart auf einer Hierarchieebene agieren. Eine zwischenmodulare Kommunikation bzw. Nachbarsensitivität ist nur in Sonderfällen wie bspw. bei dem Ausfall oder der 
Übergabebereitschaft von Produkten zu berücksichtigen. Das MTP erlaubt die Hierarchisierung von Modulen, setzt sie aber nicht voraus.

A5 - Anmelde- und Identifikationsmechanismen: Für ein Plug \& Produce ist es neben der flexiblen Ansteuerung wichtig, dass sich die Module selbstständig an dem Orchestrierungssystem anmelden, sich identifizieren, die Zugriffsschnittstelle teilen und ihre Dienste preisgeben. Dies ist sowohl bei dem MTP als auch in der CP Factory bis auf die Kopplung zwischen GM und AM nur manuell möglich.

A6 - Bedarfsorientierte Steuerung: Damit die individuellen Eigenschaften der in der Fertigungstechnik herrschenden Produktvarianz berücksichtigt werden können, sollen die Module wie bei der CP Factory in der Lage sein, die Produkte zu identifizieren. Für jedes identifizierte Produkt sollen dann die korrekten Dienste mitsamt den hinterlegten Parametern ausgeführt werden - die Identifikation und der Bedarf der Produkte soll somit der Auslöser der Dienste sein. Das MTP trifft keine Aussagen über die Notwendigkeit der Identifikation der Produkte, dies könnte jedoch in einem Dienst abgebildet werden. Prinzipiell werden Dienste per rezeptbasierter Steuerung umgesetzt, können aber auch bedarfsorientiert ausgeführt werden, sofern das Orchestrierungssystem dies unterstützt.

A7 - Produktion bei Kommunikationsabbruch: In der CP Factory und bei dem MTP kommunizieren die Steuerungen der Module über eine direkte Verbindung mit einem Orchestrierungssystem. Dies soll auch hier zutreffen. Sofern die Kommunikation aufrecht erhalten bleibt, funktionieren die Module einwandfrei. Sobald die Kommunikation abbricht, ist die Ausführung der Produktionsprozesse in den meisten Fällen abhängig von der Dienstart nicht mehr möglich. Damit die Module weiterhin produktiv sind, sollen die Module die Produktion bei einem Ausfall der zentralen Steuerung oder der Kommunikation aufrechterhalten.

A8 - Einheitliches Informationsmodell: Für die Integration von Modulen ist es wesentlich, dass die Orchestrierung erfährt, wie die Module zugreifbar sind und welche Dienste mit welchen Parametern zur Verfügung stehen. Diese Informationen sollen in einem einheitlichen und standardisierten Informationsmodell und Format vorliegen. Das entspricht dem MTP, was bei der CP Factory in einem proprietären Format unvollständig zur Verfügung steht.

A9 - Weiterführende vernetzte Eigenschaften: Über die automatisierungstechnischen Informationen hinaus, sind für die Auswahl von Modulen und die korrekte Handhabung weiterführende Eigenschaften wie z.B. die Geometrie oder die Hardware-Anschlüsse im Informationsmodell notwendig. Die Informationen sollen vernetzt sein und sich gegenseitig referenzieren, sodass eine domänenübergreifende Modellierung von Modulen möglich ist. Diese Art der Information und der Verbindungen ist bislang im MTP als auch bei der CP Factory nicht verfügbar.

A10 - Virtuell-reale Kopplung: Für die VIBN sollen die virtuellen und realen Module gegenüber der Orchestrierung auf identische Weise behandelt werden. Die Module werden damit auf dieselbe Weise in ein Anlagenverbund integriert und orchestriert. Das hat den Vorteil des effizienten Austauschs und Tests neuer und unbekannter Module im Kontext einer realen Anlage. Das MTP trifft keine Aussagen über die Integration virtueller Module, wobei die Schnittstelle der Module unabhängig davon ist, ob die Module real oder virtuell vorliegen. Die CP Factory ermöglicht die reine VIBN der Module über ein proprietäres Simulationstool, wobei ein gemischter Betrieb nicht untersucht wurde.

\section{Konzepte für das Plug \& Produce}

Die Vision von METHODS besteht darin, Plug \& Produce von modularen heterogenen Fertigungsanlage mit gemischt real-virtuellem Engineering zu ermöglichen. Das Konzept sieht gemäß Bild 1 vor, die Steuerungen der Module mit einer flexiblen Steuerungsarchitektur (A) für das Plug \& Produce auszustatten. Damit einhergehend werden umfassende Informationsmodelle der Module (B) erstellt, die als Teilmodell(e) in Industrie 4.0 Verwaltungsschalen (VWS) [6] integriert (D) werden. Dabei wird die erfolgreiche Methodik des MTP der Prozessautomation erstmalig auf die Domäne der diskreten Automatisierung übertragen. Aus der Anforderungsanalyse ist zu entnehmen, dass das MTP aufgrund seines

Open Access. (c) 2022 P. Habiger, G. Hildebrandt, R. Drath, M. Barth, A. Fay, A. Zor und M. Marseu This work is licensed under the Creative Commons Attribution 4.0 License. + (cc) Br-sA 
herstellerneutralen Einsatzes einen Großteil der Anforderungen erfüllt. Es ist jedoch nicht für die fertigungsspezifischen Anforderungen wie die bedarfsorientierte Ansteuerung konzipiert, sodass die konkrete Adaption geprüft werden muss. Mittels der domänenübergreifenden Anwendung des MTP wäre sogar die Interaktion von prozesstechnischen, fertigungstechnischen und logistischen Modulen und Anlagen möglich. Zur Erleichterung des Modul- und Anlagenengineerings sollen zudem reale und virtuelle Fertigungsmodule durch ein neuartiges virtuelles Engineering direkt miteinander interagieren können (C). Daraus resultiert, dass der Entwurf, die Visualisierung, der Test und die Inbetriebnahme virtueller Module im Kontext der realen Anlage stattfinden kann [7]. Durch die VWS werden Module im Netz find- und erkundbar und in einer dafür geeigneten Industrie 4.0 Plattform (E) automatisch vom übergeordneten Orchestrierungssystem (F) erkannt. Diese ist somit in der Lage die Module zu explorieren, zu einem geeigneten Anlagenlayout zu orchestrieren und anzusteuern. In diesem Beitrag liegt, wie bereits beschrieben, der Fokus auf den ersten drei Architekturbestandteilen (A - C) und repräsentiert damit die Grundlage für das heterogene, real-virtuelle Engineering sowie für Lehrkonzepte des virtuellen Arbeitens im realen Kontext. Der Konzeptteil C ist für den ersten Schritt lediglich im Rahmen einer Virtuellen Inbetriebnahme umgesetzt. Die Teilkonzepte sind in den folgenden Abschnitten beschrieben.

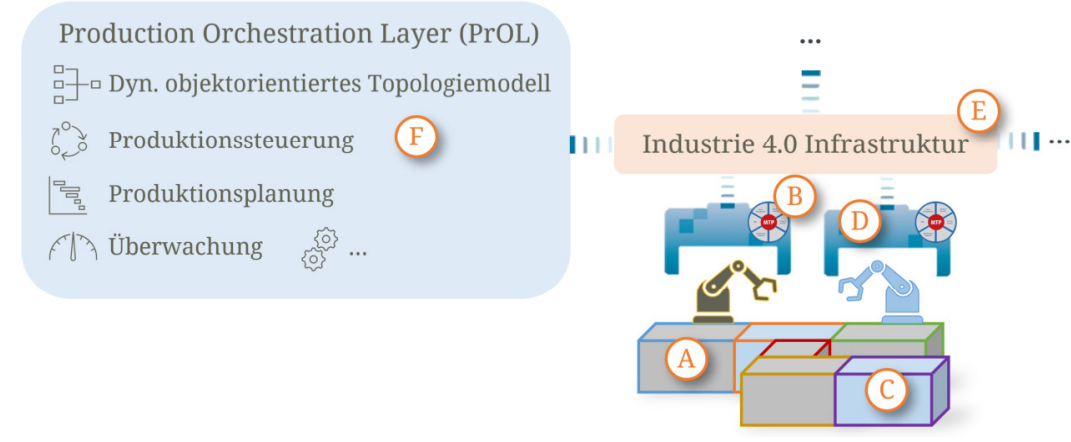

Bild 1: Vision von METHODS mit Architekturbestandteilen (A-F)

\subsection{Steuerungsarchitektur}

Aus Bild 1 ist zu entnehmen, dass die Kommunikation zwischen einem Orchestrierungssystem und den Modulen über eine VWS erfolgt. Die VWS kombiniert normierte Industrie 4.0 Schnittstellen mit Informationsmodellen. Durch sie werden die Module im Netz find- und erkundbar. Die VWS in Kombination mit dem MTP bietet in Anbetracht der hier geltenden Anforderungen großes Potential. Während das MTP die grundlegende Schnittstelle der Module definiert (A1) und diese in einem einheitlichen Informationsmodell (A8) bereitstellt, ist die VWS für Anmelde- und Identifikationsmechanismen (A5) sowie die Datenhaltung weiterführender vernetzte Eigenschaften (A9) geeignet.

Für die Steuerungsarchitektur bedeutet dies, dass die Grundfunktionalitäten in Form von MTPDiensten wie z.B. Materialtransport und -bearbeitung oder die Kommunikation zur Orchestrierung von den Modul-Steuerungen selbst übernommen werden (A2). Diese Dienste sind am MTP orientiert parallel ausführbar, können gegenseitig unterlagert sein und von überlagerten Diensten angesteuert werden und erfüllen über Prozeduren die gewünschte Variantenvielfalt aus A3. Optionale Funktionalitäten wie z.B. das Produkthandling sind hier auch möglich. Höherwertige Funktionalitäten des Plug \& Produce wie die Registrierung, Anmeldung und Authentifizierung in der Orchestrierung oder die Informationsübermittlung der Zugriffsorte, Statuswerte oder nicht-steuerungsrelevanter Informationen werden seitens der VWS ausgeführt. Die VWS bildet somit den Mittler zwischen Industrie 4.0 Anwendungen und den Steuerungen der Module. In diesem Zusammenspiel werden die Funktionen der Module auf das Minimum - die reine Ausführung der vorgesehenen Funktionalitäten - reduziert. Die Aufteilung der Funktionalitäten ist in Bild 2 angedeutet. 
Aus A7 ergibt sich, dass die Module direkt über ein Orchestrierungssystem angesteuert werden sollen und nicht gänzlich durch die VWS gekapselt werden. Das hat den Vorteil, dass der Ausfall der Industrie 4.0 Kommunikation und der Absturz von VWS-Servern nicht die Produktionsfähigkeit der Anlage beeinflussen. Sofern doch die Kommunikation zum Orchestrierungssystem oder dieses selbst ausfällt, soll gemäß A7 die Produktion fortgesetzt werden. Dies soll durch eine asynchrone Übertragung der Parameter für die individuellen Produkte geschehen, deren Informationen in separaten Produktinformationsmodellen hinterlegt sind. Der Auslöser der Dienste mit den passenden Parametern ist die Erkennung und Identifikation der spezifischen Produkte und deren Zustände (A6) in Form von dafür vorgesehenen Diensten. Bislang sieht das MTP nur vor, dass Parameter in bestimmten Zuständen übermittelt werden und ist nicht dafür vorbereitet, dass mehrere Parametersätze zu beliebigen Zeitpunkten für die individuellen Produkte übertragen werden.

Die VWS verwendet zur Reduzierung der Komplexität dieselbe Kommunikationsschnittstelle zum Modul wie das Orchestrierungssystem, wobei diese nicht steuernd zugreift, sondern vorwiegend lesend und schreibend für Registrierungsdaten. Alle weiteren an der Kommunikation beteiligten Systeme wie z.B. Diagnose- und Überwachungssoftware greifen über die Industrie 4.0 Plattform und die VWS auf die Modulinformationen zu. Das Orchestrierungssystem ist selbst auch über die Industrie 4.0 Plattform mit den VWS gekoppelt, sodass darüber die Zugriffschnittstelle der Module abgerufen werden kann. Die Schnittstelleninformation muss daher nicht wie bei dem klassischen MTP manuell im Orchestrierungssystem eingepflegt werden, sondern erfolgt dynamisch über die VWS.

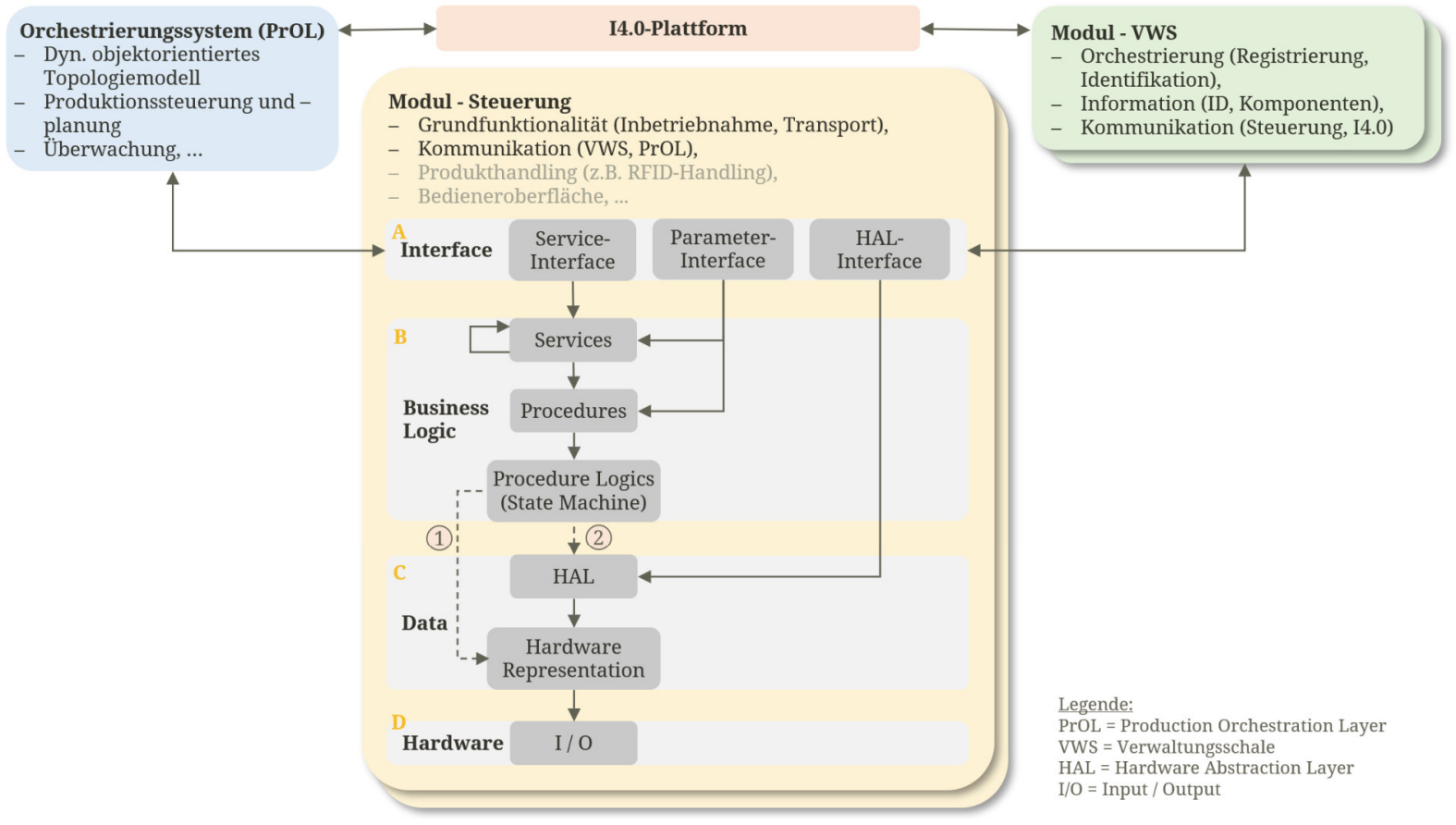

Bild 2: Abgrenzung der Steuerungsarchitektur und Ebenenhierarchie

Ein weiterer Aspekt, der in Bild 2 zu erkennen ist, ist die Vermeidung der Hierarchisierung der AM und GM der CP Factory. In METHODS werden alle Module gemäß A4 auf einer Hierarchiestufe betrachtet. Es setzt zwar durch die geringere Abstraktionsebenen voraus, dass die Kommunikationslast mit dem Orchestrierungssystem erhöht ist und darin die Kopplungslogik der Module stattfinden muss, aber birgt dennoch viele Vorteile: Alle Module werden gleichbehandelt, sie sind zentral verwaltet, lose gekoppelt und einfach auszutauschen, ohne vorher gegenseitig bekannt zu sein. 
Zusätzlich zur reinen Aufteilung der Funktionalitäten definiert METHODS eine Ebenenhierarchie (s. Bild 2), die sich an einigen Referenzimplementierungen des MTP wie z.B. die ABB-Implementierung ${ }^{9}$ und allgemeinen Steuerungsarchitekturen [8] orientiert. Die Ebenenhierarchie besteht aus vier Ebenen und der Zugriff erfolgt von oben (A) nach unten (D): Die Interface-Ebene (A) umfasst die Kommunikationsschnittstelle für die Dienste, Parameter und internen Komponenten. In der Business LogicEbene (B) sind die Funktionalitäten der Module gekapselt. Dort sind die Dienste sowie Prozeduren mit deren Implementierung inklusive dem Zustandsmodell des Dienstes enthalten. Dienste können hierarchisch aufgebaut sein. Auf der Data-Ebene (C) befindet sich die sog. Hardware Abstraction Layer (HAL) und die Hardware-Repräsentation. Die HAL dient als Adapter [9] für den Zugriff auf die Hardware-Repräsentationen (2), die in ihren proprietären Funktionsbausteinen vorliegen. Die HAL ist im MTP ursprünglich für den Zugriff der Bedienbilder konzipiert, wobei diese auch für die interne Ansteuerung verwendet werden kann. In den Fällen, bei denen die Aktoren und Sensoren keine konkrete Repräsentation in der HAL zugewiesen werden können, werden deren Hardware-Repräsentationen direkt mit den Prozeduren gekoppelt (1). Die Verbindung der Hardware-Repräsentation mit der realen Hardware (D) findet über I/O-Signale oder Feldbuskommunikation statt.

\subsection{Informationsmodellierung}

Neben der Steuerungsarchitektur findet auch die Informationsmodellierung auf Basis des MTP statt (A8). Für jeden Modultyp existiert ein MTP. Im Zusammenspiel mit einer VWS wird das MTP „als Ganzes“ in eine VWS integriert [10] und von dort aus für das Orchestrierungssystem verfügbar gemacht. Das hat den Vorteil, dass die Engineering-Daten nicht erneut reproduziert und redundante Informationen vermieden werden. Innerhalb der VWS sind weiterführende Informationen und Eigenschaften (A9) über die Module enthalten, die für eine Orchestrierung relevant sind, wie z.B. die IP-Adresse.
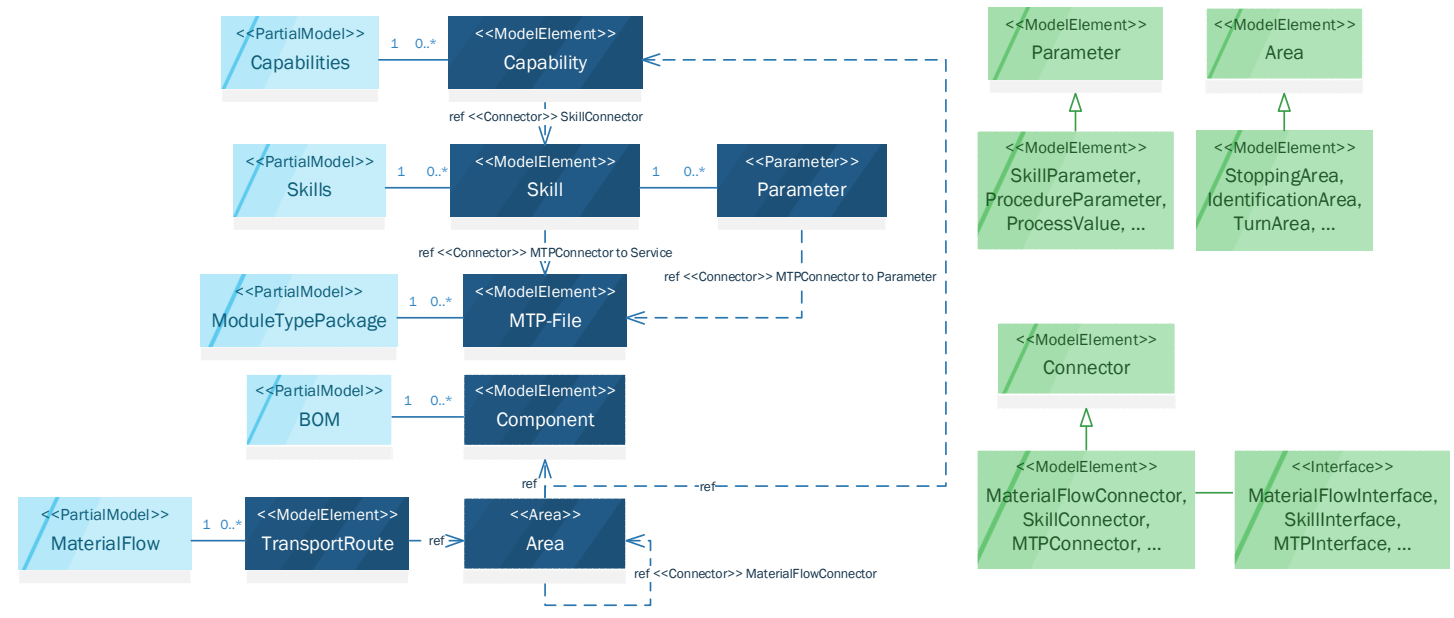

Bild 3: Auszug des Konzepts der Informationsmodellierung

Basierend auf einer ausführlichen Recherche in Abgleich mit den Anforderungen des Projektes METHODS sind folgende grundlegende Modellierungsvorschriften definiert. Die Modellinhalte werden als Teilmodelle strukturiert. Es werden möglichst viele bereits etablierte Teilmodelle, wie z.B. das Namensschild (Nameplate), verwendet. Die Teilmodelle und deren Merkmale werden über Referenzen gekoppelt. Für die domäneninterne und -externe Kopplung von spezifischen Modellelementen wie z.B. der Dienste oder des Materialflusses wird das Konnektor-Konzept aus [11] angewandt.

${ }_{9}^{9}$ ABB MTP: https://new.abb.com/control-systems/modular-automation/module-type-package 
Konnektoren sind eigenständige und semantische Modellelemente, die reale und virtuelle Schnittstellen zwischen Komponenten bilden. Es werden sowohl domänenbasierte Teilmodelle wie z.B. Commissioning und Maintenance als auch sichtenbasierte Teilmodelle wie z.B. ElectricalView oder MechanicalView für die Reduktion redundanter Informationen und Bündelung in zusammengehörige Teilmodelle eingesetzt. Eine technologieunabhängige Abbildung eines vereinfachten Teilausschnitts des Schemas des Informationsmodells ist in Bild 3 dargestellt. Im rechten Bereich sind einige Varianten von Modellelementen aufgezeigt, die für eine konkrete Modellierung notwendig sind. So gibt es bspw. unterschiedliche Arten von Konnektoren. Im linken Bereich sind einige vernetzte Informationen aufgezeigt, die über die Konnektoren und Schnittstellen in Zusammenhang stehen. Bspw. referenziert eine Fähigkeit auf einen Skill, der auf einen Service innerhalb des MTP-Dokuments verweist, das Teil des MTP-Teilmodells ist. Weiterführende Informationen zu dem Informationsmodell folgen in weiteren Publikationen.

\subsection{Virtuelle Inbetriebnahme}

Im Projekt METHODS entstehen komplexe Konzepte für das virtuelle Engineering im Kontext von realen modularen Produktionsanlagen wie zum Beispiel die Interaktion virtueller und realer Module im gemeinsamen Produktionskontext [7]. Für den Fall der reinen Ansteuerung der Module und somit der VIBN ist u.a. gefordert, dass die virtuellen und realen Module mit denselben Mitteln angesteuert werden (A10). Die in Kapitel 4.1 beschriebene einheitliche Schnittstelle ist unabhängig davon, ob das Modul real oder virtuell existiert - die Schnittstelle gegenüber der Orchestrierung und VWS ist gleich aufgebaut. Bei der Verwendung von herstellerneutralen Kommunikationstechnologien wie z.B. OPC UA merkt die Orchestrierung keine Unterschiede zwischen den virtuellen und realen Modulen. Die Schnittstelle gegenüber der Hardware (s. Ebene D aus Bild 2) kann wiederum auf individuellen Wegen wie z.B. Shared-Memory erfolgen.

\section{Prototypische Umsetzung}

Die gesamte prototypische Implementierung ist anhand des Branch-Grundmoduls der CP Factory aufgezeigt. Das reale Modul besitzt eine S7-1512 Steuerung von Siemens und das virtuelle Modul ist mit einer virtuellen SPS von CoDeSys abstrahiert. Die Module sind damit in der Lage, die Schnittstelle gegenüber der Orchestrierung und VWS per OPC UA bereitzustellen. Für die Implementierung der Steuerungsarchitektur im TIA Portal kommt die Referenzimplementierung von ORCA ${ }^{10}$ und für CoDeSys das PA-Toolkit ${ }^{11}$ von Festo SE zum Einsatz. Zur Umsetzung der Funktionalitäten des Moduls und den dazugehörigen Betriebsmitteln auf dem Modul sind die in Bild 4 orange dargestellten Dienste und Dienstparameter definiert. Bspw. wird jeder Stopper mit seiner spezifischen ID aktiviert (Release) und jede Weiche mit der Angabe des Fahrtziels mit Turn angesteuert. Des Weiteren sind die Komponenten auf die Control Modules des MTP übertragen, in Bild 4 blau dargestellt, sodass z.B. die Stopper und Weichen mit dem BinVlv abgebildet werden können. Für das RFID-Lesegerät existiert kein entsprechendes Control Module, sodass dieses derzeit nicht über die HAL abstrahiert werden kann (s. (1) bei Bild 2). Dienstinterne Abhängigkeiten sind gemäß der VDI 2658-4 berücksichtigt, sodass bspw. der Stopper nicht gleichzeitig herunter- und hochfahren kann. Bislang existiert kein Orchestrierungssystem, das die Module ansteuert, sodass dies manuell über den UA Expert stattfindet. Die Abbildung einer Produkt- und Auftragsvielfalt ist dabei schwierig abzubilden. Daher ist die Steuerung derzeit so implementiert, dass die Module noch unabhängig von den Produkt-IDs die Dienste ausführen, d.h. bei

\footnotetext{
${ }^{10}$ MTP - Moduldesign mit TIA Portal: https://support.industry.siemens.com/cs/de/de/view/109783062

${ }^{11}$ PA-Toolkit: https://www.festo.com/pa-toolkit
} 
der Erkennung eines Produktes wird immer der Parametersatz verwendet, der als letztes an der Parameter-Schnittstelle für einen Dienst zum Idle-Zeitpunkt anliegt. Die Dienste werden trotzdem intern bedarfsorientiert gestartet und gehen nach der erfolgreichen Ausführung in den Idle-Status zurück. Das Informationsmodell umfasst sowohl das für das Branch-Modul angewandte MTP als auch weitere Informationen von Bild 3. Das Modell ist als *.amlx für Automation Component [11] umgesetzt, da dieses für das Engineering von Automatisierungssystemen ausgelegt und damit einfacher zu handhaben ist als bspw. *.aasx. Die Datei wird in eine VWS „als Ganzes“ basierend auf [10] integriert.
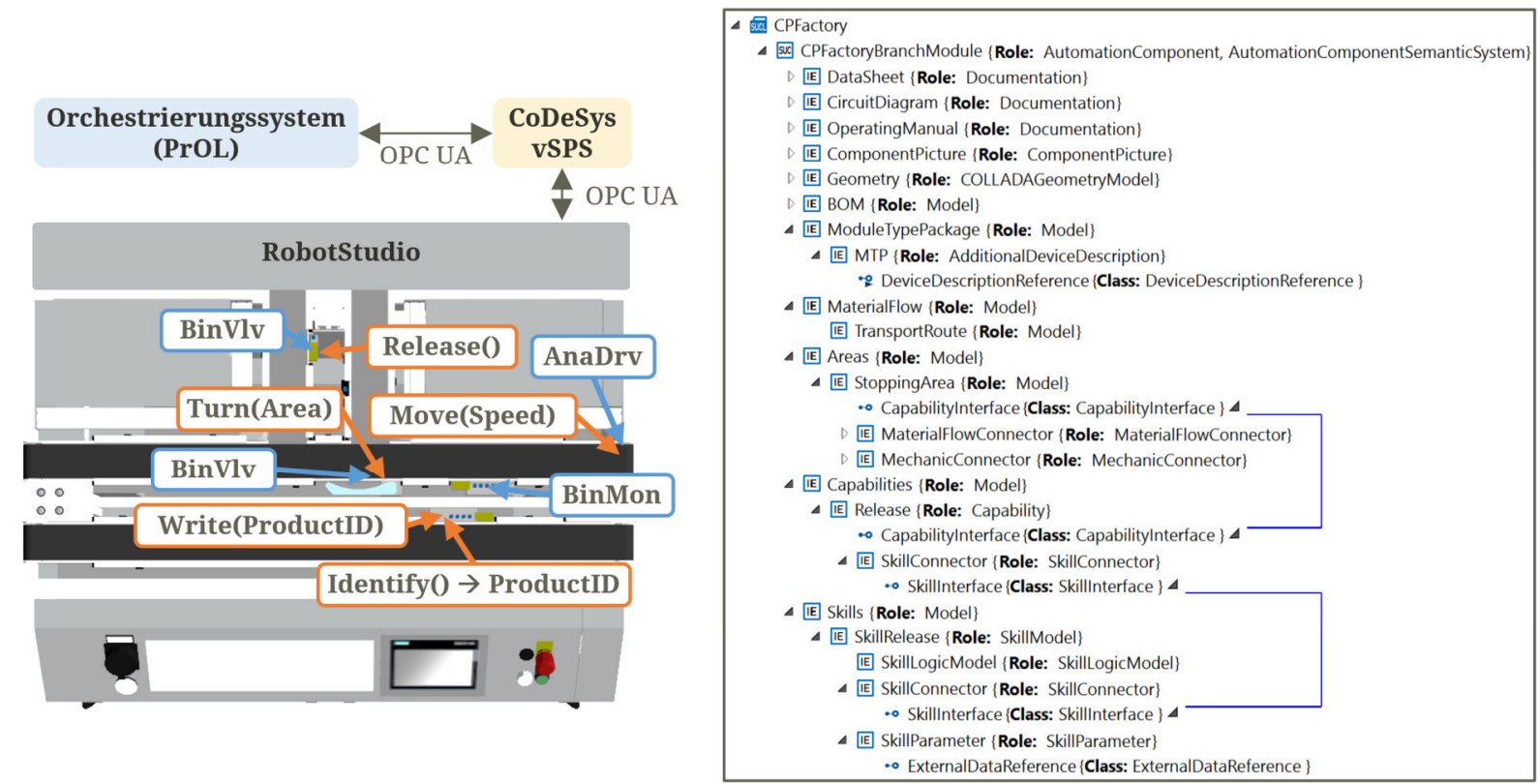

Bild 4: Dienste, Komponenten, Informationsmodell und virtuelle Inbetriebnahme

Die VIBN erfolgt exemplarisch aus dem Zusammenspiel von CoDeSys und RobotStudio (s. Bild 4). Die Module sind in RobotStudio kinematisiert, sodass die I/O-Signale der virtuellen Steuerung von CoDeSys über eine OPC UA Schnittstelle ausgetauscht werden. Die Ausführung der Dienste und Komponenten findet in der virtuellen Steuerung statt. Das bedeutet, dass die virtuelle Steuerung die Daten wie in Kapitel 4.1 beschrieben, für die PrOL bereitstellt. Das kinematisierte Modell ist auch in der Lage, das RFID-System der realen Module vereinfacht abzubilden.

\section{Ergebnisse und Ausblick}

Der Vergleich der Konzepte und der prototypischen Implementierung mit den Anforderungen an die Steuerungsarchitektur, Informationsmodellierung und VIBN für ein heterogenes Plug \& Produce zeigt einen hohen Erfüllungsgrad auf (siehe Tabelle 1), wobei einige Inhalte in der prototypischen Implementierung noch nicht untersucht wurden. Die auf dem MTP basierende dienstbasierte Steuerungsarchitektur (A2) ermöglicht den einheitlichen Zugriff (A1) auf Funktionalitäten in unterschiedlichen Varianten sowohl in Bearbeitungsmodulen als auch in Transportmodulen (A3), die allesamt an ein Orchestrierungssystem direkt gekoppelt sind (A4). Die Interaktion mit einem Orchestrierungssystem ist derzeit nur vereinfacht durch den manuellen Zugriff über OPC UA aufgezeigt. Hierzu gilt es zukünftig die Interaktion in Bezug zur bedarfsorientierten Steuerung (A6) zu überprüfen, und zu ermitteln, inwiefern die asynchrone Übertragung der Parameter zugehörig zu den identifizierten Produkten für die Sicherheit bei einem Kommunikationsabbruch (A7) hierüber realisiert werden kann. Es wird bereits ermöglicht, dass die Module die Produkte identifizieren und dadurch ausgelöst die Produktion 
starten, jedoch ist die Handhabung mehrerer Produkte in unterschiedlichen Produktkonfigurationen und -zuständen noch zu klären. Das für die automatisierungstechnische Integration relevante herstellerneutrale Informationsmodell (A8) in Form des MTP ist in einem weiterführenden Informationsmodell eingebettet, sodass diese vernetzten Informationen (A9) über die VWS abrufbar sind. Die VWS werden auch für die Anmelde- und Identifikationsmechanismen (A5) eingesetzt, wobei diese nicht in diesem Beitrag behandelt sind. Die VIBN zeigt auf, dass sich die Module sowohl in realer als auch in virtueller Form gleich gegenüber der Orchestrierung verhalten. Die Anwendung der gemischt realvirtuellen Kopplung (A10) ist bereits in [7] angedacht und wird in METHODS weiter untersucht.

Tabelle 1: Erfüllungsgrad der Anforderungen $(\checkmark=$ erfüllt, $(\checkmark)=$ teilweise erfüllt, $x=$ nicht erfüllt, $-=$ keine Angabe, ${ }^{*}=\mathrm{zu}$ prüfen)

\begin{tabular}{|c|c|c|c|c|}
\hline Nr. & Anforderung & CP Factory & MTP & METHODS \\
\hline A1 & Harmonisierte Zugriffsschnittstelle & $x$ & $\checkmark$ & $\checkmark$ \\
\hline A2 & Dienstbasierte Steuerungsarchitektur & $(\checkmark)$ & $\checkmark$ & $\checkmark$ \\
\hline A3 & Dienstvarianten & $x$ & $\checkmark$ & $\checkmark$ \\
\hline A4 & Keine Modulhierarchie & $x$ & $(\checkmark)$ & $\checkmark$ \\
\hline A5 & Anmelde- und Identifikationsmechanismen & $x$ & $x$ & $\checkmark^{*}$ \\
\hline A6 & Bedarfsorientierte Steuerung & $\checkmark$ & - & $\checkmark^{*}$ \\
\hline A7 & Produktion bei Kommunikationsabbruch & $x$ & $(\checkmark)$ & $(\checkmark)^{*}$ \\
\hline A8 & Einheitliches Informationsmodell & $x$ & $\checkmark$ & $\checkmark$ \\
\hline A9 & Weiterführende vernetzte Eigenschaften & $x$ & $x$ & $(\checkmark)$ \\
\hline A10 & Virtuell-reale Kopplung & - & - & $(\checkmark)^{*}$ \\
\hline
\end{tabular}

Zusammenfassend zeigt der Beitrag auf, dass basierend auf den Konzepten des MTP und der VWS, modulare und heterogene Produktionsanlagen für das Plug \& Produce - auch für Forschungs- und Lehrzwecke der Industrie 4.0 - realisiert werden können. Als Demonstrator bietet hierfür die CP Factory eine hervorragende Grundlage für die Konzeption und Umsetzung des erarbeiteten Ansatzes. Darüber hinaus wird die CP Factory laufend weiterentwickelt, sodass die Konzepte der Industrie 4.0 in Plug \& Produce Szenarien, Produkten und Lösungen von der Festo Didactic SE umgesetzt werden.

\section{Literatur}

[1] C. Plehn, „A method for analyzing the impact of changes and their propagation in manufacturing systems,” Dissertation, Institut für Maschinenbau, Technische Universität München, München, 2017.

[2] T. Holm, „Aufwandsbewertung im Engineering modularer Prozessanlagen,” Dissertation, Institut für Automatisierungstechnik, Helmut-Schmidt-Universität, Hamburg, 2016.

[3] S. Lier und M. Grünewald, „Net Present Value Analysis of Modular Chemical Production Plants,“ Chemical Engineering \& Technology, Bd. 34, Nr. 5, S. 809-816, 2011.

[4] J. Höll et al., ,Seamless simulation toolchain for virtual engineering and virtual commissioning of smart factories,“ in 18. Internationales Stuttgarter Symposium, Stuttgart, März 2018, S. 797-811.

[5] Automatisierungstechnisches Engineering modularer Anlagen in der Prozessindustrie - Allgemeines Konzept und Schnittstellen, VDI 2658-1, 2017.

[6] R. Drath et al., „Die Rolle der Industrie 4.0 „Verwaltungsschale“ und des „digitalen Zwillings“ im Lebenszyklus einer Anlage,“ in Automation 2017, Baden-Baden, Juni 2017.

[7] G. Hildebrandt, P. Habiger und R. Drath, „Virtual-In-The-Loop-Engineering: A categorisation and terminology for modular plants and interfaces,“ in ETFA 2021, Västerås, Schweden, Sept. 2021. 
[8] C. Lehnert et al., „A Hierarchical Domain-Specific Language for Cyber-physical Production Systems Integrating Asset Administration Shells,“ in ETFA 2021, Västerås, Schweden, Sept. 2021.

[9] A. Stutz und M. Mauermaier, „Mastering complexity with object-oriented design,“ atp edition, Bd. 60, Nr. 8, S. 34-45, 2018.

[10] S. Grüner et al., „Integration von Module Type Package und Industrie 4.0 Verwaltungsschale,“ in Automation 2021, Baden-Baden, 2021.

[11] AutomationML e.V., „AutomationML Part 6 - AutomationML Component,“ https://www.automationml.org/download-archive/ (Zugriff am 16.11.2021). 